great planets is taken as origin, and the motion calculated by mechanical quadratures. The final residuals of Pluto are all less than $4^{\prime \prime}$, which is not unduly large, as in many cases the images were ill-defined and far from the centre of the plate. The period is 248.43015 years, and the eccentricity $0 \cdot 2486438$ (it is an aid to memory that the first three digits in these two elements are the same); the perihelion passage is on Sept. 30, 1989. There will not be a conjunction with Neptune at minimum distance ( 3 units) for about eight thousand years.

The determination of Pluto's mass from Neptune depends largely on the two observations made by Lalande in 1795. As he observed the Right Ascensions to the nearest half-second of time only, they are not exact enough to give a precise result ; it is shown that a mass of Pluto, $\mathbf{1 . 3}$ times the earth's, gives a zero residual in 1795 , while a zero mass makes the 1795 residual $9^{\prime \prime}$. It will, however, be possible to derive a fair value of Pluto's mass from its recent approach to Neptune, when the latter has been observed for some eighty more years, as its orbit will then be known independently of Lalande's positions. Dr. Bower sug gests that the approach to Uranus in 1968 (distance 13 units) should also be utilised; for this purpose a long series of accurate positions of Uranus, especially from 1940 to 2000 , should be obtained. He suggests photography, with the magnitude of Uranus reduced to that of the comparison stars.

The only estimates of Pluto's mass that can be made at present are based on its stellar magnitude and apparent diameter; these both suggest a mass smaller than that of the earth. However, the unexpectedly high value for the mass of Triton, recently found at Mt. Wilson, suggests a similar possibility for Pluto. M. Baldet, as the result of visual observations with the Meudon equatorial, concludes that the diameter does not exceed $0 \cdot 2^{\prime \prime}$ (Report of the Paris Observatory for 1930).

Dr. Bower's article contains an accurate ephemeris of Pluto from August 1931 to June 1932; this is for the equinox of 1900 ; Dr. Bower notes that this is the most convenient equinox if one uses positions of the comparison stars derived from the astrographic catalogues; these contain fainter stars than those given in other catalogues. The images of brighter stars on the Pluto plates are too large for accurate measurement.

\section{A New System of Film Projection.}

W

ELL-ATTENDED demonstrations were given at the premises of Messrs. Robinson, King and Co., Ltd., in Stratford, London, of an invention by Dr. R. T. A. Innes, formerly Union Astronomer, Johannesburg, for increasing plasticity and saving space in projection of films. In place of the usual screen a large plane mirror is to be installed, the picture being projected on an ordinary screen so situated that the audience sees it in the mirror. It may be behind them, or on the ceiling. In the demonstration it was behind, the projector being behind and above the mirror.

Regarding the increase in plasticity claimed, we must confess that we failed to perceive it as regards black and white films. It is also claimed that the effects of grain and scratches are masked, but the films shown were not worn; a slight diminution in the sparkle due to grain was perhaps noticeable. An excellent coloured film shown was, however, certainly improved. Colour adds plasticity to films, and Dr. Innes's plan really 'varnishes' the picture, as it were, and thus adds somewhat to the brilliance and depth of the colour. The effect is similar to the difference between matt and glazed photographs, and would finally, as in this case, be a matter of taste. The lure of devices of this kind for inventors is due to the psychological fact that any change in the tint or texture of a photograph, if it is novel, is at first pleas. ing, since the photograph is inherently inadequate as a representation of three-dimensional reality. The change is usually imagined to make the picture more real-to fulfil our unconscious wish : this will be re. membered when the tinting of films was first introduced, and it accounts for the plastic effect of colour. The unsophisticated are immensely pleased by an extremely brilliant glaze on photographs. But these joys are fleeting: custom soon stales them.

The other advantages claimed are more substantial. Installed in a small room or hall, practically the whole floor space may be filled with seats from which a good view is obtained, since even those close to the mirror see the screen as at a convenient distance. Those at the back have a more distant view than they otherwise would, but can still see well. Thus the available space is much increased. The field for the invention is thus chiefly in small, cramped halls, and in education and advertising.

Difficulties arise from the fact that mirrors exceeding a certain size are expensive to make and handle. Even a small screen would need to be built up of two or three parts, and means would have to be found to make the join unobjectionable. Also, the film is seen reversed if projected upon the screen in the usual way, while serious loss of light occurs with through projection, apart from the fact that the gain in space would no longer be present. With on-projection, therefore, the film has to be turned round in the projector, and few projectors allow of this being done with soundfilms, since it brings the sound track on the wrong side. However, one portable equipment at least is available in which this difficulty does not occur, and with such an apparatus and Dr. Innes's mirror a small room could be packed with spectators who would all get a satisfactory view.

\section{University and Educational Intelligence.}

Cambridge.-Dr. F. C. Phillips, of Corpus Christi College, has been appointed University lecturer in petrology.

In a report to the University on forestry in the University curriculum, the General Board states that there is practically no future for graduates trained in forestry except in Government forest services. Only twenty to twenty-five of these posts are available each year, and there are no fewer than five university schools, including that at Cambridge, training candidates for these posts. As a result of investigations, the Committee of the General Board concludes that the University is not justified in maintaining a forestry organisation as a recruiting ground for Government services, partly because the demand is so small, but even more because the Committee considers that University policy in forestry teaching cannot be recon. ciled with the present official view. It is accordingly recommended that the Department of Forestry be suppressed, examinations in forestry for the ordinary B.A. degree be discontinued after 1934, and examinations for the diploma in forestry cease in October 1935.

ShEFFIELD.- The Council of the University has made the following appointments : Mr. B. H. Bentley, lecturer in botany, to be professor of botany; Dr. R. N. Rudmose Brown, lecturer in geography, to be professor of geography; Mr. L. E. S. Eastham, to be professor of zoology; Dr. J. Florey, to be professor of pathology; and Dr. J. W. Edington, to be professor of bacteriology.

No. 3242, VoL. 128] 
Aт the Indian Institute of Science, Bangalore, the service of Dr. M. O. Forster as director has been extended until April 1933. Dr. V. Subrahmanyan has been appointed professor of biochemistry.

AT the end of the year Dr. R. S. Clay is resigning the principalship of the Northern Polytechnic, London, a post he has occupied for twenty-nine years. The governors, staff, students, and members of clubs and societies who have been associated with him during this long period wish to assist in offering some token of their regard and affection, and a committee has been set up to give effect to this general desire. The honorary treasurer of the committee is Mr. W. M. Macbeth, Northern Polytechnic, Holloway, London, N.7.

THE International Federation of University Women has issued a report of its sixteenth council meeting, held at Wellesley College, Massachusetts, last April under the chairmanship of the president of the Federation, Prof. Winifred Cullis, and attended by women graduates of thirty-one countries. Prominent among the activities of the Federation is the establishment of research fellowships to give university women the opportunity of a year's work in a foreign country. The fund for this purpose already exceeds five thousand pounds, exclusive of the collections of the American Association of University Women, which will be allocated partly to national and partly to international fellowships.

\section{Birthdays and Research Centres.}

Dec. 20, I876.-Dr. W. S. AdAMs, director of Mount Wilson Observatory.

My chief interest for many years has consisted in physical and quantitative studies of stellar spectra. Theoretical astrophysicists have carried on much able research during recent years on problems of stellar constitution and radiation, and the need for observational evidence to aid in discriminating among different theories is pressing. This is especially true of investigations dealing with temperatures and pressures in stellar atmospheres, the opacity coefficient, and the abundance of the elements in different states of excitation and ionisation.

Specific observational studies at Mount Wilson are dealing with the character, intensities, and contours of stellar spectral lines, their differential displacements, and the application of spectral criteria to the determination of the absolute brightness and the distances of stars.

Dec. 22, I 862.-Dr. VAUGHAN CoRnISH, past president of Section E (Geography) of the British Association and of the Geographical Association.

The secret of scenic beauty is the enhancement which landscape derives when the grouping happens to be harmonious, whether this be a matter of locality, as when the physical features are harmonious, or of season, as when light or colour are happily blended or contrasted.

During the last six years, I have published a number of original papers in which the principle of harmonious grouping has been applied both to the landscape of the wild and to the scenery of civilisation, and in a chapter entitled "Watching the Seasons" in a recently published book on "The Poetic Impression of Natural Scenery "I have shown its application to the changes of sunlight and foliage.

Every advance in the systematic study of natural beauty will be a social benefit, since an enhanced appreciation of the countryside will provide the majority of our people with a corrective for the inevitable artificiality of urban life.

\section{Societies and Academies.}

\section{LONDON.}

Royal Society, Dec. 10.-Sir Arthur Eddington : On the mass of the proton. From the theory of the cosmical constant developed in a previous paper, a wave equation for an electron is developed, which, however, is incomplete. The correct equation contains the factors 10 and 136 , representing the number of degrees of freedom associated with the respective energy terms. The mass of a particle satisfying this wave equation is given by an equation having two roots, which evidently correspond to electrons and protons, and their ratio is $1847 \cdot 60$. It is verified that the two roots represent charges of opposite sign.P. S. H. Henry : The specific heats of air, oxygen, and nitrogen from $20^{\circ} \mathrm{C}$. to $370^{\circ} \mathrm{C}$. Further developments of the new constant flow calorimeter for the determination of the specific heats of gases at constant pressure are given. These results, whilst agreeing with those obtained by the sound velocity method at room temperature, show a much higher rate of increase of the specific heats with temperature, and a closer approximation to the curves predicted by means of Boltzmann's hypothesis from the spectroscopic frequencies of vibration of the molecules.-W. $\mathrm{L}$. Francis: Studies in membrane behaviour (1). Equilibrium membrane potentials are measured for a buffer concentration gradient of $10: 1$ using $N / 40-N / 400$ and $N-N / 10$ solutions. The e.m.f.'s with and without gelatine on the membranes are compared over the $p \mathrm{H}$ range $2 \cdot 35-7 \cdot 3$. The results, more particularly with weaker solutions, appear to support the modified diffusion theory, that is, the anions are retarded in basic solution and the cations in acid solution. Direct evidence, given by determinations of the transport numbers of the sodium and acetate ions across the membrane in $N / 2$ solution, vetoes this theory. Assuming that the membrane potentials are made up of the ordinary diffusion potential and an opposed e.m.f. due to the combination or adsorption of ions on the protein, an explanation is suggested which conforms with the known behaviour of gelatine in electrolyte solutions.

Paris.

Academy of Sciences, Nov. 9.-E. Jouguet: The secular stability of the rotors of turbines. A discussion of the criteria of stability according to Stoidola's hypotheses, with the additional criterium suggested by Lamb.-H. Vincent: A theory of the constitution of antibodies. A comparison of the properties of antitoxins and cryptotoxins (toxin + sodium salicylate). Both form a stable complex with the toxin and both are dissociated when the $p \mathrm{H}$ is lowered to $4.7:$ although the toxin is neutralised, it is not destroyed in either case.-André Blondel: The mutual inductances of magnetic leaks in transformers with several secondaries.-Erik Westzynthius: The distribution of integers which are not divisible by any one of the $n$ smallest prime numbers.-A. Marchand : Various extensions of the idea of a continuum of limited order.-J. Favard : A proposition of Minkowski.-S. Finikoff : Stratifiable parabolic congruences: transformers of $R_{0}$ surfaces.- J. Herbrand and C. Chevalley: New demonstration of the theorem of existence in the theory of the body of classes.-H. Parodi : The method of integration by successive arcs giving, in the calculation of the elementary arc, an approximation as close as desired.-Georges Giraud : Problems of values at the boundary in the case of discontinuous data.-J. A. Lappo-Danilevski: The construction of the normal integral matrix of a system of linear differential equations in the neighbourhood of a pole of its coefficients.-A. Markoff : A general property

No. 3242, Vou. 128] 